\section{Meninges Type Tumors}

Mi-Yeoung Jo

Independent Practice, Sherman Oaks, CA, USA

\section{Synonyms}

Meningeal tumors

\section{Definition}

Meninges type tumors arise from the meninges (dura mater, arachnoid, pia mater) covering the brain and the spinal cord. There are three common types of meningeal tumors, classified according to tumor grade. Grade I meningiomas are the most common of the meningeal tumors and are usually slow growing and benign. They typically do not invade the brain parenchyma. Surgical resection of this type of tumor usually results in good prognosis. Grade II and III meningeal tumors (e.g., atypical, chordoid, clear-cell, papillary, and anaplastic) occur infrequently and are more infiltrative and malignant. They can also metastasize to other parts of the body. Grade I meningeal tumors are more common in women, while grade II and III meningeal tumors are more common in men. Symptoms often arise from pressure and compression rather than brain infiltration, with more specific symptoms depending on the location of the tumor.

\section{Cross-References}

Meninges

Meningioma

Tumor Grade 